possible that antipsychotic medication was the initial intervention used and the patient took it as a matter of routine.

In summary, medication adherence is a complex issue that can be affected by various factors, such as lack of insight, religious and cultural beliefs, level of education and socioeconomic status, comorbid alcohol misuse, to name a few. ${ }^{4}$ We believe further studies are needed in this area.

1 Perecherla S, Macdonald AJD. Older psychiatric in-patients' knowledge about psychotropic and non-psychotropic medications. Psychiatrist 2011; 35: 220-4

2 Mitchell AJ, Selmes T. Why don't patients take their medicine? Reasons and solutions in psychiatry. Adv Psychiatr Treat 2007; 13: 336-46.

3 Droulout T, Liraud F, Verdoux H. Relationships between insight and medication adherence in subjects with psychosis. Encephale 2003; 29: 430-7.

4 Patel MX, David AS. Medication adherence: predictive factors and enhancement strategies. Psychiatry 2007; 6: 357-61.

Gurinder P. Singh, CT2 psychiatry, Health Lane Hospital, West Bromwich, Black Country Partnership NHS Foundation Trust, email: gurinder.singh@bcpft.nhs.uk; Aparna Prasanna, consultant old age psychiatrist, Black Country Partnership NHS Foundation Trust, Wolverhampton.

doi: $10.1192 / p b .36 .5 .195 a$

Authors' response: We agree that adherence to medication is important and subject to complex influences. We thought that understanding of medication was a neglected factor and set out to study this rather than adherence. We had hoped that this was clear. We were surprised to find that, broadly speaking, patients understood psychotropic and nonpsychotropic medication to the same degree. We confirm that patients from ethnic minorities who were able to speak English were included; patients were in acute wards and not long-stay wards (of which we have none). In the example of how we chose which medication to ask about, we do not say that we selected the mood stabiliser over the antipsychotic because it was given first. We chose it because it was likely to be used for the longest time. We agree that our sample was not representative of all older psychiatric patients and say as much in the discussion.

Sri Perecherla is consultant psychiatrist at Woodcote House, Croydon University Hospital, email: sri.perecherla@slam.nhs.uk. Alastair J. D. Macdonald is visiting professor of psychiatry in the Health Services and Population Research Department, Institute of Psychiatry, London.

doi: $10.1192 / \mathrm{pb} .36 .5 .196$

\section{Recruitment in psychiatry}

Those concerned about the dearth of young doctors applying to train as consultant psychiatrists might usefully consider the motives of those who make this choice. I am a recently retired general adult consultant psychiatrist that worked in England. I chose to undertake training because I wished to emulate senior consultants whom I met while acting as a medical student or junior doctor. I admired their determination and aspiration to improve the lives of those suffering from serious mental illness and their central role in the clinical care of those referred to mental health services. However, I fear junior doctors will now find it difficult to meet such inspirational and dynamic clinicians.

In England the blame culture consequent on the repeated internal, coroner and external enquiries, reconfiguration of services, the provisions of the amended Mental Health Act and New Ways of Working for consultants psychiatrists (and others) have all undermined morale. This last development left me without responsibility for my in-patients, the autonomy to arrange urgent admission when I thought this necessary or, in some cases, to refer for appropriate psychological therapy. Working became an increasing challenge. Our junior doctors notice these developments and their effect on senior colleagues' attitudes. It does not surprise me that the number opting to train remains worryingly low.

Keith E. Dudleston, consultant psychiatrist (retired), Ivybridge, email: tp@rcpsych.co.uk.

doi: $10.1192 / p b .36 .5 .196 a$

\section{Retaining trainees in psychiatry through a more mindful practice}

Barras \& Harris's survey on retention difficulties in psychiatry ${ }^{1}$ in provoking further discussion about the state of psychiatric training is an important piece of work. The systemic effects of the current economic crisis cannot be separated out from implications to services and in turn their impact is felt by those working and training within the system. Having myself recently completed higher training in psychotherapy, and through my experience of facilitating trainee case-based discussion groups, many of the trainees' comments picked up by Barras \& Harris felt all too familiar.

In terms of trainee concerns over the attitude of others towards psychiatry, I very much agree with the thinking of the authors that better integration of psychiatry with other specialties may increase understanding of both the contribution of psychiatry and challenge of mental health difficulties. Alongside this, I also think it is important to recognise that to bear with the projected 'madness' of others, which may mean we are seen as unsettling and to be kept a distance from perhaps by devaluation, is an important function of psychiatrists. Trainees' function as containers can be fostered, for example, in case-based discussion groups, enabling them to begin to understand and tolerate some of these processes as they are played out in their day-to-day work.

In Barras \& Harris's study, when asked about work and patient care, trainees complained about too much paperwork and a pressure to appear to be 'doing things correctly', which both undermine the real patient care. The concept of social defence, as described by Menzies-Lyth in her study of poor medical nursing staff retention in hospitals, ${ }^{2}$ is helpful in thinking about some of these difficulties. In mental hospitals, working practices which reduce contact with patients, such as the care of an individual patient being split into tasks or reduplication of checks to eliminate or share the responsibility of decisions, are used by staff/managers because of a fear of being in contact both with patients' and their own 'mad violence' and fragmentation. Further to this, the additional pressures of restructuring may both add to and be part of the same process. Ballatt \& Campling in Intelligent Kindness ${ }^{3}$ remind us that 'there is certainly evidence that major structural change keeps senior managers and board members detached from the front line of healthcare' (p. 131). In the face of this poor containment by the organisation, it is not surprising that morale is low among trainees. 
The Royal College of Psychiatrists' Faculty of Medical Psychotherapy has recently been working towards an education strategy for the renewal and development of a more psychotherapeutic psychiatry, with the aim of bringing psychotherapy to the heart of psychiatry. I think that the model of meaning and mind that psychotherapy brings to the practice of psychiatry is crucial in enabling us to work with our disturbed patients, and as such it should be embedded into training.

1 Barras M, Harris J. Psychiatry recruited you, but will it retain you? Survey of trainees' opinions. Psychiatrist 2012; 36: 71-7.

2 Menzies-Lyth I. Containing Anxiety in Institutions. Free Association Books, 1988

3 Ballatt J, Campling P. Intelligent Kindness: Reforming the Culture of Healthcare. RCPsych Publications, 2011.

Meave T. Fingleton, locum consultant medical psychotherapist, The Pines Psychotherapy Service, Coventry and Warwickshire Partnership NHS Trust, Warwick, email: meave.fingleton@nhs.net

doi: $10.1192 / \mathrm{pb} .36 .5 .196 \mathrm{~b}$

\section{MRCPsych CASC exam: is there a better choice?}

The basic intention behind the final membership exams is to test the abilities and competencies of the candidates at the consultant level. An ideal exam offers equal chance and challenge to all its candidates. It checks both elementary and higher knowledge in all the necessary domains of the subject. A final membership exam should ideally be exhaustive, with an intrinsic ability to test the range, width and depth of the candidate's knowledge.

The Royal College of Psychiatrists' exams have been globally known for their excellence and high standards. MRCPsych Paper 1, 2 and 3 check the theoretical knowledge of the candidate, but owing to the complex nature of mental disorders, a psychiatrist needs to have a much wider and in-depth understanding rather than mere theoretical knowledge of the subject. The Clinical Assessment of Skills and Competencies (CASC) mainly appears to be good at testing the communication skills, mannerisms, body language and the ability of a candidate to handle a situation. This is clearly reflected in the high passing rates in CASC of graduates from the UK and other countries where English is the primary language compared with candidates from other countries. ${ }^{1}$ If we look at the passing rates of the practical components of the old MRCPsych Part 2 (i.e. individual patient assessment and patient management problems), we will see that the gap between the passing rates of UK graduates (including candidates from countries with English as their first language) and of the candidates from other countries is much narrower. ${ }^{2}$

To make matters worse for overseas candidates, there are so many variables in the present CASC exam that it is almost impossible for the candidates to understand where they are faltering. Some candidates seem unable to tell why they passed or why they failed. This has caused a lot of anxiety, discouragement and frustration, leading to a feeling of helplessness among overseas candidates who repeatedly fail at this exam despite scoring highly in their theory exams. On the other hand, CASC has its own advantages of being a fair deal to all examinees, covering various subspecialties at the same time, checking the ability of a candidate to handle a difficult clinical situation or for that matter the ability to control the interview. The local candidates seem to have an undue advantage when it comes to testing these skills. Being born and brought up in the UK (or in countries with English as the first language) gives them an upper hand when it comes to testing the doctor-patient interaction. For candidates whose primary language is not English, passing this exam seems to be comparatively a much more difficult task. In an attempt to 'perform a task in 7 minutes or 10 minutes in an artificial situation', the 'performer' just 'spits' out whatever he/she has crammed up, although in a sophisticated and palatable manner. There is no time to think, understand, plan or use any innovative strategies utilising the vibrantly balanced bio-psychosocial model embedded in the spirit of psychiatric management.

This is the final exam and we are testing the basic skills such as overdose, psychopathology and Mini-Mental State Examination (MMSE). The old-style MRCPsych exam Part 2 had great advantages. It presented the examinees with complex psychiatric situations (patient management problems) and offered them a chance to think, analyse, innovate and use a multidimensional biopsychosocial model when faced with questions directly from the consultants who had a much deeper knowledge in that field. It had the ability to analyse and evaluate the in-depth understanding of the system and the ways in which psychiatry works. The examinee's attitude, competence and excellence could then be gauged in the right way. Old-style exams carried a great advantage of being 'face to face' viva with consultants (rather than with an actor and consultant being a silent watcher as in (ASC) and provided a much more robust assessment of the quality and range of the candidate's knowledge at both academic and pragmatic levels. Few disadvantages of the old style examination were that it involved the real patient (individual patient assessment) who might have been slightly drowsy or restless due to side-effects of medications and it was very difficult to standardise as candidates often saw different patients.

There can never be an ideal exam and people will always have complaints, but if we combine the present CASC style with the old style of MRCPsych exam, we can have the best of both.

Considering the highly acclaimed quality of exams conducted by the UK medical Royal Colleges, it is time to reconsider and realise that the exam should not put any of the candidates at a disadvantage just because of their language and be equally challenging not only to people whose primary language is not English but also to people who have a limited knowledge of psychiatry. It would be a fair and balanced exam if instead of having 16 CASC stations there were 8 CASC stations and 8 stations of patient management problems/vivas so that it could provide candidates with a platform to prove their substance in both domains, namely communication and a thorough knowledge of the functioning of psychiatry as a system. Therefore, if two of the above are integrated, it may come closer to an ideal exam.

1 Bateman A. MRCPsych Examinations Cumulative Results 2008-2010. Royal College of Psychiatrists, 2011.

2 Oyebode F, Furlong E. MRCPsych examinations: cumulative results 1997-2002. Psychiatr Bull 2007; 31: 61-4.

Gursharan Kashyap, MBBS, DCH, MD, MRCPsych, ST4 trainee in general adult psychiatry, South Essex Partnership University NHS Foundation Trust (SEPT), email: gursharan.kashyap@sept.nhs.uk; Akeem Sule, MRCPsych, consultant psychiatrist, SEPT.

doi: 10.1192/pb.36.5.197 